\title{
Surface field excitation by an obliquely incident wave
}

\author{
I. V. Konoplev, ${ }^{1}$ A. R. Phipps, ${ }^{2}$ A. D. R. Phelps,${ }^{2}$ C. W. Robertson, ${ }^{2}$ K. Ronald, ${ }^{2}$ \\ and A. W. Cross ${ }^{2}$ \\ ${ }_{1}^{1}$ JAI, Department of Physics, University of Oxford, Oxford OXI 3RH, United Kingdom \\ ${ }^{2}$ SUPA, Department of Physics, University of Strathclyde, Glasgow G4 ONG, United Kingdom
}

(Received 28 February 2013; accepted 25 March 2013; published online 10 April 2013)

\begin{abstract}
Observation of surface field excitation by an obliquely incident wave inside a cylindrical two-dimensional periodic surface lattice is a step forward toward $\mathrm{THz}$ Cherenkov amplifiers. Here we observe and discuss this phenomenon, investigating it using different approaches. The results are compared, and it is shown that an increase in the lattice contrast results in excitation of surface fields by an obliquely incident wave resulting in the eigenmode formation. Measurements were conducted by studying forward transmission of the signal, and it is shown that the measured gaps in the spectra are associated with the eigenmode formation rather than band gap establishment. (C) 2013 AIP Publishing LLC. [http://dx.doi.org/10.1063/1.4801759]
\end{abstract}

Development of high-power amplifiers of coherent radiation capable of producing multi-watt output power in the sub-millimeter wavelength range is one of the challenging problems. Such devices are required for weather radar applications, for tracking nano-satellites and space debris, as well as for plasma diagnostics, volcano monitoring, and quality control of pharmaceutical products, and for the study of historical artefacts. ${ }^{1-3}$ In recent work, ${ }^{4-6}$ it has been suggested that periodic patterns machined on the inner surface of a cylindrical conductor exhibit properties of a thin dielectric layer allowing such phenomena as Cherenkov instabilities to be observed. In particular this is an attractive possibility for creating high-intensity sources of $\mathrm{THz}$ radiation driven by mildly relativistic electron beams, as such structures should allow very effective heat, stress, and electric charge management via their quick dissipation into the main body "sink" of the material. Conventionally the surface periodic structures previously considered ${ }^{4-8}$ were low contrast (the corrugation amplitude is less than a quarter of the operating wavelength) and were used as distributed mirrors and high-Q cavities or low contrast interaction regions to mediate a Cherenkov instability. ${ }^{7,8}$ Until now it has only been demonstrated that coupling between a wave at normal incidence and a surface field has been possible, ${ }^{4}$ thus effectively denying the possibility of applying such structures to design an amplifier. In this paper we can now demonstrate that coupling between a "near grazing," obliquely incident wave and a surface field is possible if a high-contrast structure ${ }^{6}$ (corrugation amplitude is larger than a quarter of the operating wavelength) is used. We are studying both low and high contrast Periodic Surface Lattice (PSLs), with the ultimate goal of designing a lattice exhibiting the properties that can enable it to support broadband amplification in the $\mathrm{THz}$ frequency range, demonstrating the possibility of tailoring artificial structures for a specific need. Indeed we are planning to exploit the main advantages of such an artificial structure to design an Amplifier of Coherent Radiation (ACR). One can define the structure built from a good conducting metal (like copper) showing dielectric properties on its surface as a "topological conductor" in contrast to the well-known "topological insulator." The 2D surface patterns improve mode stability while mediating coupling between the specific surface and propagating volume fields, thus bringing the possibility of maintaining the radiation coherence and observing the input signal amplification. In this paper we present the results of numerical and experimental studies of a cylindrical 2D PSL with a range of contrasts and designed as an initial "proof of principle" in the W-band $(75 \mathrm{GHz}-110 \mathrm{GHz})$ frequency range (Fig. 1(a)). We show that increase of the lattice contrast results in the appearance of coupling between the oblique (near-grazing) volume wave and the surface field resulting in excitation of an eigenmode of the PSL.

The cylindrical 2D PSL shown in Figure 1 has been created by machining sinusoidal radial variations onto an aluminium former

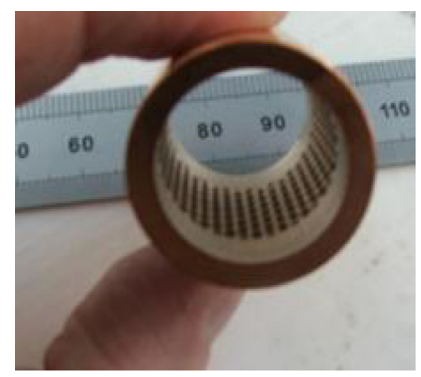

(a)

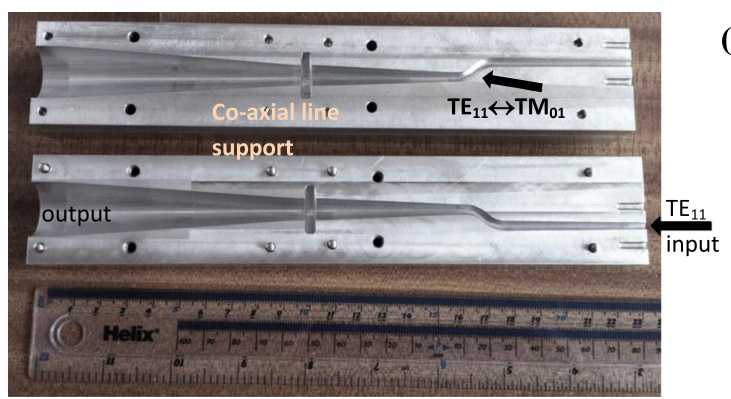

(b)

FIG. 1. (a) Photograph of the 2D PSL machined on the inner surface of a copper cylinder and having mean radius $r_{0}=1 \mathrm{~cm}$; amplitude of corrugations $\Delta r=0.5 \mathrm{~mm}$; number of azimuthal periods 20; and longitudinal period $3 \mathrm{~mm}$. (b) Photograph of the experimental setup designed to measure 2D PSLs as shown above. 


$$
r=r_{0}+\Delta r \cos \left(\bar{k}_{z} z\right) \cos (\bar{m} \varphi) .
$$

Here $r_{0}=10 \mathrm{~mm}$ is the unperturbed radius of the cylindrical waveguide, $\Delta r$ is the corrugation amplitude, $\bar{k}_{z}=2 \pi / d_{z}$, $d_{z}=3 \mathrm{~mm}$ is the period of the perturbations in the $\mathrm{z}$-direction, and $\bar{m}=20$ is the number of azimuthal variations. Four individual aluminum formers of varying perturbation amplitude [ $\Delta r=0.25 \mathrm{~mm} ; 0.3 \mathrm{~mm} ; 0.5 \mathrm{~mm} ; 0.8 \mathrm{~mm}$ ] were constructed, in order to investigate the effect of the amplitude of the perturbations on the coupling of the surface and volume fields. Copper was electroformed onto the surface of the formers, and once machined to the required radius the aluminum was removed by dissolving it in a strong alkaline solution. This leaves the copper structure with the perturbations on the inside wall. The structure with the highest amplitude of perturbations $0.8 \mathrm{~mm}$ is a high contrast case, as its peak-to-peak corrugation of $1.6 \mathrm{~mm}$ (from top to bottom of the grating) is larger than a quarter of the operating wavelength. In Figure 1(a) the photograph of the structure with $\Delta r=0.5 \mathrm{~mm}$ is shown. All lattices were machined to have 7 full longitudinal periods $\left(d_{z}\right)$ each with a $5 \mathrm{~mm}$ section of smooth cylindrical waveguide at the ends to ensure good connection between the lattice and the experimental set up. Let us note that the parameters of the lattices studied are close to ones which are planned to be used to observe ACR based on a Cherenkov interaction. The large diameter of the structure will allow high current (low current density) electron beam transportation while avoiding RF breakdown, structure overheating, beam disruption, and parasitic mode generation. To study such a structure using a wave-beam consisting of a single mode, an apparatus made from a single block of aluminium was designed and constructed (Figure 1(b)). The apparatus is different, from the conventional set-ups such as Kretschmann-Raether and Otto configurations. ${ }^{9}$ It was developed to excite surface fields (surface plasmons in optics) on the inner surface of the cylindrical periodic structure. This configuration allows irradiation of the cylindrical lattice at a range of angles between the wave vector and the axis of the cylinder from approximately $90^{\circ}$ (a nearly cut-off wave) to approximately $0^{\circ}$ (a propagating wave having transverse wavenumber much smaller than the longitudinal wavenumber, i.e., a "near-grazing" wave). It was also designed to be manufactured from a single block of aluminum (Fig. 1(b)) to reduce the number of connections, thus enabling us to avoid internal scattering and mode transformation via minimising the number of connections before the input of the PSL. The full length of the set-up after machining was $223.9 \mathrm{~mm}$ long with an input port of $2.6 \mathrm{~mm}$ diameter and a launching port of $20 \mathrm{~mm}$ diameter.

Numerical modelling of the electromagnetic field evolution and eigenmode excitation has been also conducted using the 3D code MAGIC to improve understanding of the experimental measurements. The 3D modelling of such structures is a challenge due to the large variation of the geometrical parameters (dimensions) and the oversized nature of the lattice structure. To optimise the use of the computer memory and the computer performance a square wave approximation was applied in the case of the low contrast model, ${ }^{4}$ while a sinusoidal corrugation has been used to simulate the high contrast lattices. To compare the profiles of the lattices in Figures 2(a) and 2(b) the $r-z$ cross-sections of the high and low contrast structures (left and right columns respectively) are shown. The insets in these figures show the typical contour plots of the azimuthal magnetic component $\mathrm{B}_{\varphi}$ of the eigenfield. The contour plots shown in Figures 2(a) and 2(b) were observed by launching an amplitude-modulated RF pulse in the $\mathrm{TM}_{01}$ mode, having a flat frequency spectrum in the frequency range from $80 \mathrm{GHz}$ to $110 \mathrm{GHz}$. The lattice response spectra were studied using wave beams formed by TEM and $\mathrm{TM}_{01}$ modes and measuring the decaying field spectra after the electromagnetic pulse fully propagated through the structure. Later in this paper we compare the spectra of eigenmodes excited inside high/low contrast structures (left/right rows) using TEM (solid line) and $\mathrm{TM}_{01}$ (dotted line) modes with the measured spectra. As will be discussed below by changing the radial index of the waveguide modes which form the input pulse the radiation angle with respect to the lattice can be effectively controlled. We note that the field structure observed inside the high contrast structure is very similar to that observed inside the low contrast lattice leading us to conclude that the theory developed in Refs. 4-6 for low contrast structures can be extrapolated (to some extent) to a high contrast structures.

To measure the properties of the lattice the electromagnetic wave-beam was formed using the set up shown in Fig. 1(b) which is capable of converting the fundamental $\mathrm{TE}_{1,1}$ mode of a cylindrical waveguide into either the $\mathrm{TM}_{0,1}$ mode of a cylindrical waveguide or the TEM mode of a coaxial waveguide. The wave-beam was then launched via either a co-axial line termination plane launcher (such that the lattice

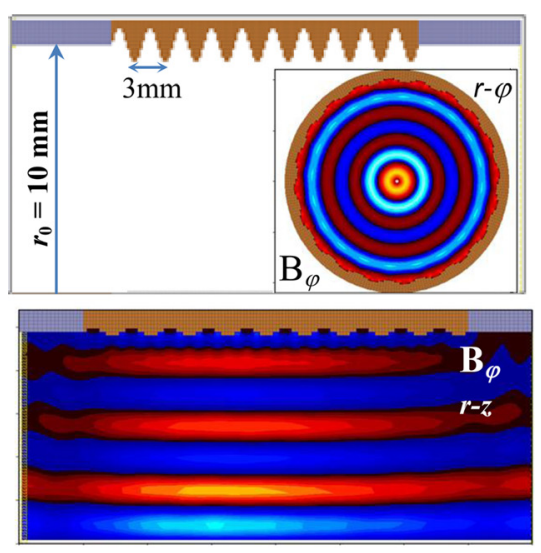

(a)

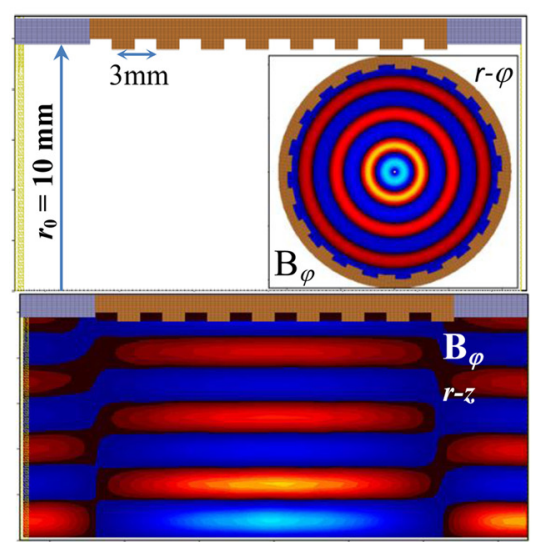

(b)

FIG. 2. Numerical models of the (a) high and (b) low contrast 2D PSLs showing $r-z$ cross-sections of the structures and contour plots of the eigenmodes' azimuthal magnetic field component excited, in $r-\varphi$ (inset to the first figure), and $r-z$ (second figure) cross-sections. 
is irradiated at all possible angles simultaneously) or via a directly irradiating structure with the $\mathrm{TM}_{0,1}$ wave-beam (oblique irradiation of the lattice at a small grazing angle). The termination of the coaxial line mediates the excitation of the azimuthally symmetric $\mathrm{TM}_{\text {on }}$ modes with a finite set of transverse wavenumbers (defined by the radial index $n$ ). At the maximum value of $n(n=N)$, the near cut-off wave is observed (wave vector at almost $90^{\circ}$ to the axis of the cylinder), and the structure is similar to that shown in Figures 2(a) and 2(b). The measurements of the PSLs were conducted in the far field zone $(27 \mathrm{~cm}$ from the output aperture), and millimeter-wave absorbent material was used to reduce parasitic reflections. A millimeter-wave Anritsu Vector Network Analyser (ME7808B) was used for the measurements. In the first set of experiments the structures were excited using a co-axial line termination plane launcher. The results of the measurements are shown in Figures 3(a) and 3(b). As expected the PSL eigenmode excitation was observed in both cases, i.e., for both the high and the low contrast PSLs. In Figure 3(a) the comparison of the results is shown for the low contrast lattices (corrugation amplitudes of $0.25 \mathrm{~mm}, 0.3 \mathrm{~mm}$, and $0.5 \mathrm{~mm}$ ) excited by the co-axial launcher. It is clear that the responses observed are similar, and the dependence on the amplitude of the corrugation is as expected, i.e., with increase of the amplitude of the corrugation the amplitude and the width of the minima measured grow but the centers are located at the same frequency, indicating the coupling of the same modes. In this, low contrast structure, case the eigenmode having 8 radial variations (formed with the partial $\mathrm{TM}_{08}$ mode) and having minima

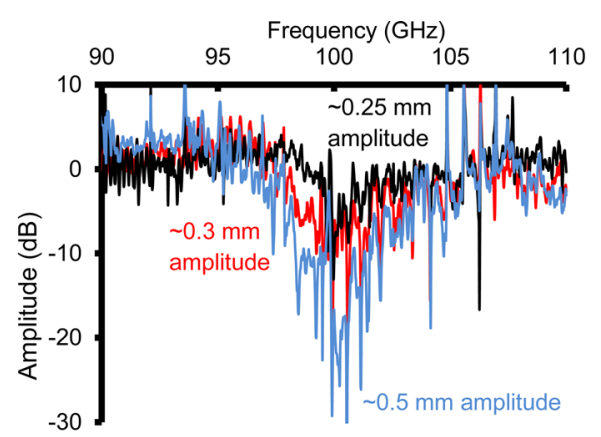

(a)

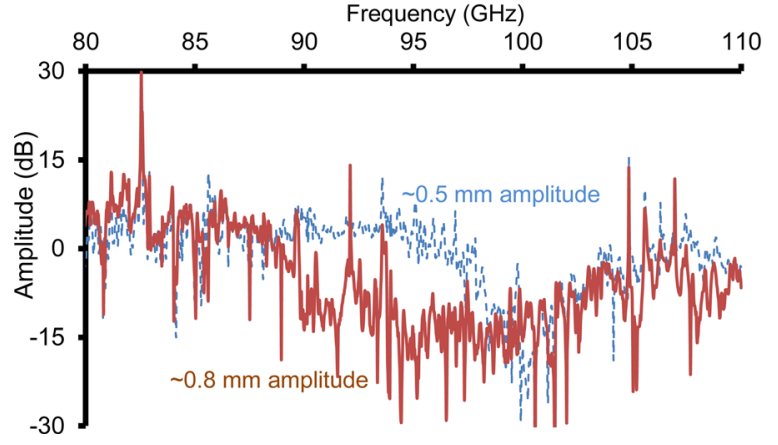

(b)

FIG. 3. Results of measurements of the electromagnetic signal transmission versus frequency (2D PSL spectral response) through (a) low (amplitude of corrugations $0.25 \mathrm{~mm}, 0.3 \mathrm{~mm}$, and $0.5 \mathrm{~mm}$ ) and (b) high (amplitude of corrugation $0.8 \mathrm{~mm}$ ) contrast structures, if the structure is radiated using co-axial launcher based on terminated co-axial line. Scattering of near cut-off (normal incidence to the surface) wave on the lattice takes place. In (b) the spectral responses of the high (solid line) and the low (broken line) contrast structures are also compared. around $100 \mathrm{GHz}$ was excited. However, when the PSL with $0.8 \mathrm{~mm}$ corrugation amplitude was measured a different behavior was observed (Fig. 3(b)). The minimum of the transmission became very broad while its amplitude stayed the same or became smaller. Also, it looks like few modes are coupled, and the minima are merged together resulting in a "red-shift" of the center of the gap. Let us note that far from the resonance frequencies the measurements coincide well at high and low frequencies. However when the co-axial launcher was substituted with the direct launcher the excitation of only the high-contrast structure was observed. In Figure 4 the results of the measurements of the structures with $0.5 \mathrm{~mm}$ and $0.8 \mathrm{~mm}$ corrugation amplitude are shown. To observe the data the PSLs were excited using a $\mathrm{TM}_{01}$ wave-beam (Fig. 2). It is interesting to note that no excitation of the low contrast structure was observed in spite of the indications from the numerical predictions. This can be explained by low coupling (which is proportional to the amplitude of the corrugation) between the lattice and the fields and relatively high losses. ${ }^{10}$ Comparing with the previous data (low contrast case) we see that the position of the minima observed for the high contrast lattice is associated with the eigenmode having 7 radial variations (formed with the partial $\mathrm{TM}_{07}$ mode) and having downshifted minima around $87 \mathrm{GHz}$. We also note that the propagating mode is now coupled to the PSL eigenmode. In Figure 5 a comparison of the spectra observed from numerical modelling and experimental measurements are shown. The minima in the measured spectra correspond to excitation of structure eigenmodes which are composed of surface fields and near cut-off fields, i.e., the radiation losses are minimal and electromagnetic (EM) energy remains and dissipates inside the lattice. To study numerically the eigenfield excitation inside the PSL based structure the near cut-off partial field associated with eigenfield was monitored. The set of maxima of the spectra observed in the numerical models is associated with excitation of the near cut-off fields which have different radial indices and thus associated with different eigenmodes of the structure. The good agreement can be clearly seen from the figures, while small deviations can be attributed to the tolerances in the lattice manufacturing as a variation of the lattice mean radius $\Delta r_{0} \sim 0.1 \mathrm{~mm}$ will result in the measured frequency shift.

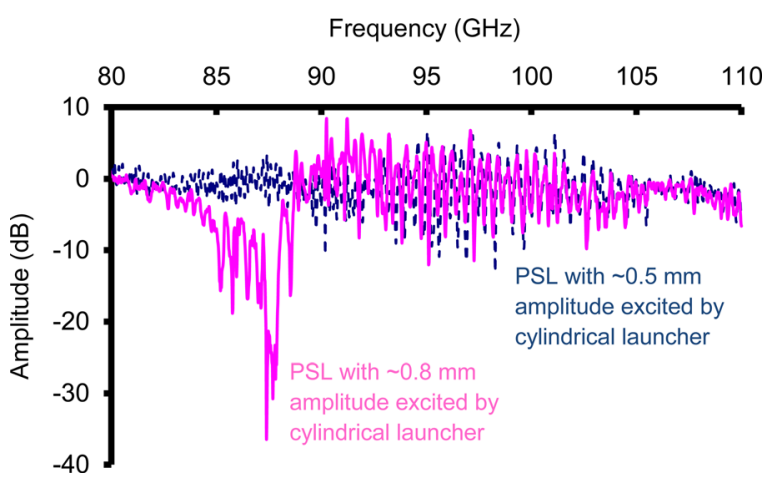

FIG. 4. Results of measurements of the electromagnetic signal transmissions versus frequency (2D PSL spectral response) through low (broken line) and high (solid line) contrast 2D PSL. The structures are irradiated by an oblique (near-grazing) incident wave-beam formed by the $\mathrm{TM}_{01}$ mode of the cylindrical waveguide. 

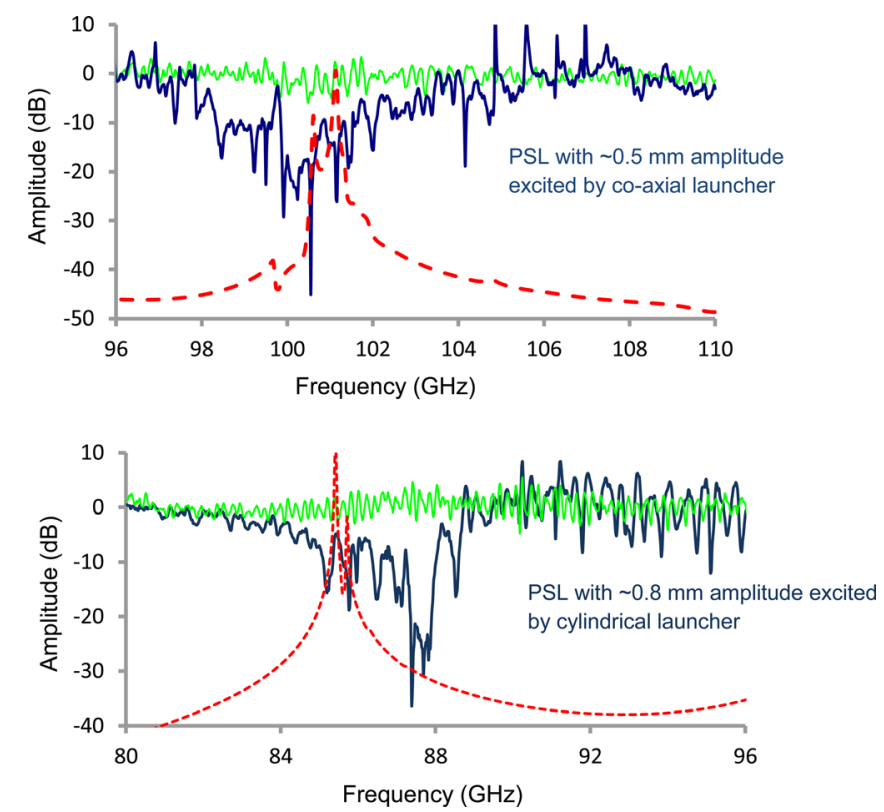

FIG. 5. Comparison of the experimental data (solid bold line) showing spectral gaps in the signal transmissions and theoretical predictions (dashed line) of 2D PSLs' eigenmodes excitation for (a) low and (b) high contrast 2D PSLs. In both figures the thin lines show the measured lattice responses if the amplitude of the corrugations is zero.

In this paper we presented numerical and experimental studies of low and high contrast two-dimensional surface periodic structures of cylindrical topology. We have shown that coupling between an oblique, near-grazing TM wave and a PSL is possible if the amplitude of the corrugation is large (high contrast structure). The coupling was not observed for low contrast structures in spite of the fact that the numerical modelling indicates the possibility of such a coupling. One of the possible explanations is that coupling is weak compared with the losses (diffraction and ohmic) inside the structure. ${ }^{10}$ Also, such losses are more difficult to take into account in numerical studies, whereas they are always present in real experiments. We note that we demonstrated the coupling between a propagating mode and the eigenmode of the PSL via the scattering of near cut-off and surface partial fields leading to the possibility of radiation synchronisation from an oversized beam in devices such as slow-wave Cherenkov amplifiers, thus allowing such devices capable of amplifying a $\mathrm{THz}$ input signal to be designed and investigated. We also have studied the dependence of the PSL spectral response on the amplitude of the corrugations, and the results have been presented and discussed. Using the 3D code MAGIC we have illustrated that if no losses are considered the eigenmode structures inside the lattices, as well as the spectral response of the structures of different contrast to excitation with an electromagnetic pulse having a flat spectrum in the frequency range $80 \mathrm{GHz}-110 \mathrm{GHz}$, are similar. The results of the experimental measurements have been presented and compared with the results from the numerical simulations, and a good agreement between the numerical models and the experimental measurements has been shown. We note that the structures studied were designed to develop $\mathrm{THz}$ amplifiers as the lack of such amplifiers significantly restricts the development of a number of applications in the medical and biological sciences and in the pharmaceutical and security industries.

${ }^{1}$ J. F. Federici, B. Schulkin, F. Huang, D. Gary, R. Barat, F. Oliveira, and D. Zimdars, Semicond. Sci. Technol. 20, S266 (2005).

${ }^{2}$ E. Abraham, A. Younus, A. El Fatimy, J. C. Delagnes, E. Nguéma, and P. Mounaix, Opt. Commun. 282, 3104 (2009); J. B. Jackson, J. Bowen, G. Walker, J. Labaune, G. Mourou, M. Menu, and K. Fukunaga, IEEE Trans. Terahertz Sci. 1, 220 (2011).

${ }^{3}$ G. L. Carr, Nature (London) 420, 153 (2002).

${ }^{4}$ I. V. Konoplev, L. Fisher, A. W. Cross, A. D. R. Phelps, K. Ronald, and C. W. Robertson, Appl. Phys. Lett. 97, 261102 (2010); A. W. Cross, I. V. Konoplev, K. Ronald, A. D. R. Phelps, W. He, C. G. Whyte, N. S. Ginzburg, N. Yu. Peskov, and A. S. Sergeev, ibid. 80, 1517 (2002); A. W. Cross, I. V. Konoplev, A. D. R. Phelps, and K. Ronald, J. Appl. Phys. 93, 2208 (2003)

${ }^{5}$ J. B. Pendry, L. Martín-Moreno, and F. J. Garcia-Vidal, Science 305, 847 (2004); I. V. Konoplev, L. Fisher, A. W. Cross, A. D. R. Phelps, K. Ronald, and C. W. Robertson, Appl. Phys. Lett. 96, 261101 (2010); A. Pors, E. Moreno, L. Martin-Moreno, J. B. Pendry, F. J. Garcia-Vidal et al., Phys. Rev. Lett. 108, 223905 (2012).

${ }^{6}$ I. V. Konoplev, A. J. MacLachlan, C. W. Robertson, A. W. Cross, and A. D. R. Phelps, Phys. Rev. A 84(1), 013826 (2011); Appl. Phys. Lett. 101, 121111 (2012).

${ }^{7}$ T. S. Chu, F. V. Hartemann, B. G. Danly, and R. J. Temkin, Phys. Rev. Lett. 72, 2391 (1994); N. S. Ginzburg, A. A. Kaminsky, A. K. Kaminsky, N. Yu. Peskov, S. N. Sedykh, A. P. Sergeev, and A. S. Sergeev, ibid. 84, 3574 (2000); I. V. Konoplev, P. McGrane, W. He, A. W. Cross, A. D. R. Phelps, C. G. Whyte, K. Ronald, and C. W. Robertson, ibid. 96, 035002 (2006).

${ }^{8}$ T. Tajima and M. Cavenago, Phys. Rev. Lett. 59, 1440 (1987); K. Okamoto, T. Noma, A. Komoto, W. Kubo, M. Takahashi, A. Iida, and H. Miyata, ibid. 109, 233907 (2012); A. Schilke, C. Zimmermann, P. W. Courteille, and W. Guerin, ibid. 106, 223903 (2011); I. V. Konoplev, L. Fisher, K. Ronald, A. W. Cross, A. D. R. Phelps, C. W. Robertson, and M. Thumm, Appl. Phys. Lett. 96, 231111 (2010).

${ }^{9}$ A. V. Zayats, I. I. Smolyaninov, and A. A. Maradudin, Phys. Rep. 408, 131 (2005).

${ }^{10}$ I. V. Konoplev, A. W. Cross, A. D. R. Phelps, and K. Ronald, Phys. Rev. E 68, 066613 (2003). 